\title{
Concentration of Plasmodium falciparum gametocytes in whole blood samples by magnetic cell sorting enhances parasite infection rates in mosquito feeding assays
}

Isaie J. Reuling, Will J. R. Stone, Marga van de Vegte-Bolmer, Geert-Jan van Gemert, Rianne Siebelink-Stoter, Wouter Graumans, Kjerstin Lanke, Teun Bousema ${ }^{\dagger}$ and Robert W. Sauerwein ${ }^{*}$

\begin{abstract}
Background: Mosquito-feeding assays are important tools to guide the development and support the evaluation of transmission-blocking interventions. These functional bioassays measure the sporogonic development of gametocytes in blood-fed mosquitoes. Measuring the infectivity of low gametocyte densities has become increasingly important in malaria elimination scenarios. This will pose challenges to the sensitivity and throughput of existing mosquitofeeding assay protocols. Here, different gametocyte concentration methods of blood samples were explored to optimize conditions for detection of positive mosquito infections.

Methods: Mature gametocytes of Plasmodium falciparum were diluted into whole blood samples of malaria-naïve volunteers. Standard centrifugation, Percoll gradient, magnetic cell sorting (MACS) enrichment were compared using starting blood volumes larger than the control (direct) feed.

Results: MACS gametocyte enrichment resulted in the highest infection intensity with statistically significant increases in mean oocyst density in 2 of 3 experiments ( $p=0.0003 ; p \leq 0.0001 ; p=0.2348$ ). The Percoll gradient and standard centrifugation procedures resulted in variable infectivity. A significant increase in the proportion of infected mosquitoes and oocyst density was found when larger volumes of gametocyte-infected blood were used with the MACS procedure.

Conclusions: The current study demonstrates that concentration methods of $P$. falciparum gametocyte-infected whole blood samples can enhance transmission in mosquito-feeding assays. Gametocyte purification by MACS was the most efficient method, allowing the assessment of gametocyte infectivity in low-density gametocyte infections, as can be expected in natural or experimental conditions.
\end{abstract}

Keywords: Malaria, Gametocytes, Mosquito-feeding assay, MACS

\section{Background}

Malaria transmission is mediated by sexual stage parasites (gametocytes) that are generated at low frequency from their asexual progenitors. Mature gametocytes are

\footnotetext{
${ }^{*}$ Correspondence: Robert.Sauerwein@radboudumc.nl

'Teun Bousema and Robert W. Sauerwein contributed equally to this work

Department of Medical Microbiology, Radboud University Medical Center, Geert Grooteplein 28, Microbiology 268, 6500 HB Nijmegen, The Netherlands
}

ingested by mosquitoes, activating inside the mosquito gut to form micro- (male) and macro- (female) gametes. Gametes sexually reproduce to form oocysts, inside which infectious sporozoites develop that make the mosquito infectious to humans once they reach the mosquito salivary glands.

Transmission-reducing interventions form essential components of global efforts to contain anti-malarial resistance [1] and eliminate malaria [2]. Such interventions will lower malaria transmission by reducing 
gametocyte production and carriage, or by interfering with sporogonic development in the mosquito. Mosquito-feeding assays are important tools to guide the development and support the evaluation of transmission-blocking interventions. These functional bioassays measure the transmission of gametocytes to mosquitoes, by allowing mosquitoes to feed either on blood from infected individuals (the direct skin or direct membranefeeding assay) [3] or cultured gametocytes (the standard membrane-feeding assay; SMFA), before assessing the infection rate in the mosquitoes by oocyst or sporozoite detection [4]. Higher densities of gametocytes are associated with higher mosquito infection rates [4], but mosquito infections may occur at very low gametocyte densities $[5,6]$. The stochastic nature of mosquito infections at low oocyst densities makes it difficult to judge whether non-infectiousness of gametocyte-positive blood samples arises because: (i) gametocytes are too low in density [7]; (ii) too few mosquitoes were examined; or (iii) other factors influencing gametocyte infectiousness (e.g. parasite factors $[8,9]$ such as maturity $[10,11]$ or sex ratio $[12,13]$. or extrinsic factors such as human or mosquito immunity [14-16]).

Measuring the viability of low gametocyte densities becomes increasingly important in malaria elimination scenarios as low density infections with gametocytes may be very common [17]. Under such conditions sporadic mosquito transmission events may be highly relevant for control efforts but will require a large number of mosquitoes to be examined.

As field studies are subject to many uncontrolled and likely confounding parameters, a controlled human malaria infection (CHMI) model for transmission from man to mosquito would be a great asset for the evaluation of (transmission-blocking) drugs or vaccines [18, 19], (Reuling et al. pers. Comm..). Here, different gametocyte concentration methods in blood samples were explored with the aim to optimize conditions for detection of positive mosquito infections.

\section{Methods}

\section{In vitro gametocyte culture}

A semi-automated suspension culture was used for the gametocyte culture with slight modification of descriptions by Ponnudurai et al. [20]. Mature gametocytes of Plasmodium falciparum (NF54 strain) were cultured in a 'shaker' flask for periods of 16 days at $37{ }^{\circ} \mathrm{C}$ under $4 \%$ $\mathrm{CO}_{2}, 3 \% \mathrm{O}_{2}, 93 \% \mathrm{~N}_{2}$ continuous gas flow. Parasites were cultured in RPMI 1640 with L-glutamine (Life Technologies), supplemented with 5.94 g/L HEPES (BDH Biochemical), $50 \mathrm{mg} / \mathrm{L}$ hypoxanthine (Sigma Aldrich), and $50 \mathrm{mM}$ of $\mathrm{N}$-acetyl glucosamine (Sigma Aldrich) to treat asexual parasites from day 8. Culture medium was changed twice daily, with an initial $4-5 \%$ haematocrit.

\section{Membrane-feeding assays}

Cultured gametocytes were diluted into whole blood samples. Venous blood of malaria-naïve volunteers was drawn into heparin-containing tubes, and kept warm in a $37{ }^{\circ} \mathrm{C}$ heat-block. Cultured $P$. falciparum gametocytes were added to the whole blood at concentrations between 300 and 1000 gametocytes/ $\mu \mathrm{L}$ (in standard experiments). Gametocytes concentrated by the various purification methods were all reconstituted in $400 \mu \mathrm{L}$ of blood, of which $300 \mu \mathrm{L}$ was transferred to an artificial membrane feeder and offered for exactly $15 \mathrm{~min}$ to a cage of 50,1-3day old Anopheles stephensi mosquitoes. The remaining $100 \mu \mathrm{L}$ of blood was stored in RNAprotect Cell Reagent (Qiagen, Hilden, Germany). Control mosquitoes were fed the gametocyte-enriched whole blood directly (without concentration). Blood-fed mosquitoes were kept on glucose at $26{ }^{\circ} \mathrm{C}$ and $70-80 \%$ humidity; on day 7 post infection the mid-guts of 20 mosquitoes in each feed group were removed by dissection, stained with mercurochrome, and examined for the presence of oocysts by microscopy.

\section{Gametocyte concentration}

All procedures were conducted at $37^{\circ} \mathrm{C}$ and samples were kept at this temperature prior to feeding to prevent early gametocyte activation. Three purification methods were compared by using starting blood volumes 5 times higher than the control (direct) feed, to assess their suitability to retain or improve infectivity in the SMFA. Because only $300 \mu \mathrm{L}$ of the $400 \mu \mathrm{L}$ concentrated feed material was fed to mosquitoes, the remainder being used for gametocyte quantification, the SMFA sample contained $75 \%$ of the total gametocyte content of purification output (equivalent to a 3.75 -fold higher start volume than the control).

\section{Standard centrifugation}

Gametocytes were separated from uninfected erythrocytes and asexual parasites by standard centrifugation causing gametocytes to form a layer on top of the erythrocytes. Gametocyte-infected whole blood was spun down at $2000 \mathrm{RPM}$ at $37{ }^{\circ} \mathrm{C}$ for $5 \mathrm{~min}$. Two-hundred $\mu \mathrm{L}$ of the top-layer of the pellet of erythrocytes was added to $200 \mu \mathrm{L}$ of plasma to adjust the haematocrit level (1:1 erythrocyte/plasma ratio).

\section{Percoll gradient separation}

Gametocytes in the infected whole blood sample were concentrated by a $63 \%$ Percoll density gradient (Percoll, GE, USA) centrifugation step by centrifugation at 2000 
RPM (3 min acceleration; 3 min break) at $37^{\circ} \mathrm{C}$ for $20 \mathrm{~min}$ $[21,22]$. The interface containing gametocytes was separated using a blunt needle, and washed twice with warm incomplete medium (RPMI 1640 parasite medium without $10 \%$ serum) at 2000 RPM for 5 min (1 min acceleration; 1 min break). The supernatant was removed leaving $50 \mu \mathrm{L}$, in which the pellet was re-suspended and mixed with $350 \mu \mathrm{L}$ of uninfected whole blood.

\section{Magnetic cell sorting (MACS) for gametocyte enrichment}

Gametocytes in the infected whole blood sample were concentrated by magnetic cell sorting (MACS) using a QuadroMACS ${ }^{\mathrm{TM}}$ separator and LS MACS columns (MiltenyiBiotech, UK) as described previously, with slight modifications [23, 24]. A 21-gauge (21G) needle $(0.8 \times 50 \mathrm{~mm})$ was attached to the column. After equilibration with $1 \mathrm{~mL}$ of warm incomplete medium, gametocyte-infected whole blood was added and the column was washed with $3 \mathrm{~mL}$ of warm incomplete medium. Next, the column was then removed from the magnet, and placed in a $15-\mathrm{mL}$ centrifuge tube and $1 \mathrm{~mL}$ of warm $\left(37^{\circ} \mathrm{C}\right)$ incomplete medium was added to the column. When the flow ceased, another $1 \mathrm{~mL}$ of medium was added to the column, and gently pressed through the column with the corresponding plunger. The gametocyteenriched suspension was spun down in a $37^{\circ} \mathrm{C}$ centrifuge for $10 \mathrm{~min}$ at $2000 \mathrm{RPM}$. The supernatant was removed leaving $50 \mu \mathrm{L}$, in which the pellet was re-suspended. $350 \mu \mathrm{L}$ of uninfected whole blood was added to the pellet and kept at $37^{\circ} \mathrm{C}$ until use. The entire MACS procedure was carried out in a heated cabinet incubator.

\section{Quantitative reverse transcriptase polymerase chain reaction}

Quantitative reverse transcription PCR (qRT-PCR) was performed targeting Pfs 25 mRNA that is specific to female gametocytes [40]. One-hundred $\mu \mathrm{L}$ of the samples (gametocyte enriched or control) were added to $400 \mu \mathrm{L}$ of RNAprotect Cell Reagent (Qiagen, Hilden, Germany) for automated extraction and qRT-PCR as described previously [25] with two modifications: $2 \mu \mathrm{L}$ cDNA was used in the $20 \mu \mathrm{L}$ final reaction mix and primer concentration was reduced to $225 \mathrm{nM}$.

\section{Statistical analysis}

Statistical analysis was performed using GraphPad Prism software version 5 (GraphPad Software Inc., California, USA). Differences in oocyst intensity between feeds were determined by the Mann-Whitney U test, and Fisher's exact test was used to test for differences in oocyst prevalence.

\section{Results}

\section{Comparison of gametocyte concentration methods}

Three independent experiments were conducted to compare oocyst intensities between standard centrifugation (Centrifugation), Percoll gradient separation (Percoll), and magnetic cell sorting (MACS) (Fig. 1).
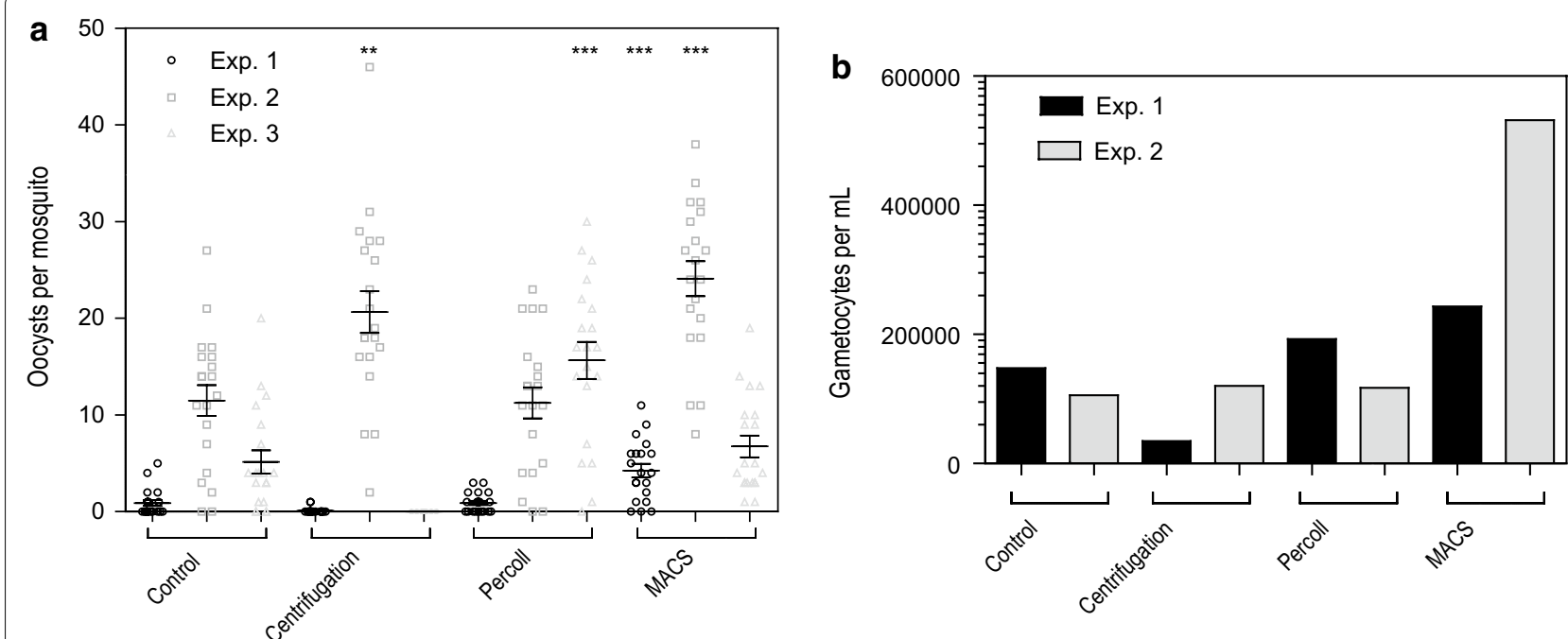

Fig. 1 Infectivity of gametocyte-infected whole blood samples after different concentration methods. a The starting material of gametocyteinfected whole blood was $300 \mu \mathrm{L}$ for the controls, which was fed to mosquitoes directly. For all non-control feeds, starting material was $1500 \mu \mathrm{L}$ of the same gametocyte-infected whole blood. Gametocytes from this starting material were concentrated, eluted and reconstituted in $400 \mathrm{\mu L}$ of uninfected blood, from which $300 \mu \mathrm{L}$ was fed to the mosquitoes. Vertical bars indicate mean with SEM. ${ }^{* *} \mathrm{p}<0.01,{ }^{* * *} \mathrm{p}<0.001$. b Gametocyte density per sample from feeding material measured by Pfs25 qRT-PCR from experiments 1 and 2 
MACS gametocyte enrichment resulted in statistically significant increases, relative to control, in mean oocyst density in 2 of 3 experiments, and numerically higher in the third experiment $(p=0.0003 ; p \leq 0.0001$; $p=0.2348$ ) (Fig. 1a, Table 1). The effect of MACS enrichment on infection prevalence (the proportion of infected mosquitoes) was statistically significant in only 1 of 3 experiments $(p=0.0187 ; p=0.4872$; $p=0.106)$. The Percoll gradient and standard centrifugation procedures showed variable infectivity results (Fig. 1a, Table 1). The mean oocyst density ranged from $0.9-11.5,0.1-20.7,0.9-15.7$, and 4.3-24.1, for the control, standard centrifugation, Percoll, and MACS, respectively. MACS gametocyte enrichment showed a 31,378 and $110 \%$ increase in mean oocysts relative to control. Percoll gradient gave the highest oocyst density in one experiment with a $202 \%$ increase in mean oocysts relative to control, compared to MACS with $31 \%$. However, the other two experiments conducted with Percoll gradient did not increase oocyst density relative to control, while the use of MACS consistently resulted in an increased oocyst density. Gametocyte density of mosquito feed material as determined by Pfs 25 qRT-PCR from experiments 1 and 2 are shown in Fig. 1b. A procedural error occurred during the qRT-PCR sampling process of experiment 3 , resulting in loss of material. Whilst these findings are based on only two experiments, and do not reflect the proportional increase in gametocyte numbers that was expected based on input volume, they suggest a pronounced increase in gametocyte numbers by MACS.
The effect of larger blood sample aliquots on gametocyte infectivity by MACS

Next, the possibility of using larger starting volumes of infected whole blood by MACS for gametocyte enrichment was examined. The infectiousness of gametocytes purified from 300, 1500 and $4500 \mu \mathrm{L}$ of gametocyteinfected blood was assessed in two separate experiments (Fig. 2a). The control direct feed with $300 \mu \mathrm{L}$ of gametocyte-infected whole blood resulted in a mean of 0.5 (range $0-3$ ) and 2.2 (range 1-7) oocysts per mosquito in two separate experiments. MACS gametocyte enrichment conducted with $300 \mu \mathrm{L}$ of purified blood gave respective mean oocyst densities of 0.95 (range $0-8$ ), and 0.35 (range 0-2) in the two independent experiments; when using $1500 \mu \mathrm{L}$, mean oocyst density increased to 2.8 (range 0-9) and 2.25 (range 0-7). Finally, using $4500 \mu \mathrm{L}$ of blood increased the mean number of oocysts to 11.1 (range 11-11.25), reflecting a 5- to 22.5 -fold difference, compared to the controls. Gametocyte density of all mosquito feed material as determined by Pfs 25 qRTPCR is shown in Fig. 2b.

\section{Enhancing infectivity of low gametocyte densities by MACS}

MACS gametocyte enrichment at low gametocyte densities was studied with the gametocyte density in culture material of $\sim 400$ gametocytes $/ \mu \mathrm{L}$ and in titration series from 1 to 6400 gametocytes $/ \mu \mathrm{L}$. The gametocyte content of the 4500 and $9000 \mu \mathrm{L}$ start volumes was thus equivalent to 11.25- and 22.5-fold higher start volumes than the control. While the control feed showed no infection of

Table 1 Details of the comparison of concentration methods. $n / N=$ Infected mosquitoes/total number of mosquitoes dissected

\begin{tabular}{|c|c|c|c|c|c|c|c|c|}
\hline Experiment & Protocol & $\begin{array}{l}\text { Blood volume } \\
(\mu \mathrm{L})^{\mathrm{a}}\end{array}$ & $\begin{array}{l}\text { Total } \\
\text { oocysts }\end{array}$ & $\begin{array}{l}\text { Infected mosq. } \\
\mathrm{n} / \mathrm{N}(\%)\end{array}$ & $p$ value* & $\begin{array}{l}\text { Mean oocysts } \\
\text { (range) }\end{array}$ & $\begin{array}{l}\% \text { change in mean } \\
\text { oocyst to control }\end{array}$ & $p$ value $*$ \\
\hline \multirow[t]{4}{*}{1} & Control & 300 & 18 & $9 / 20(45 \%)$ & - & $0.9(0-5)$ & - & - \\
\hline & Centrifugation & 1500 & 2 & $2 / 20(10 \%)$ & 0.031 & $0.1(0-1)$ & -88.9 & 0.0112 \\
\hline & Percoll & 1500 & 18 & $11 / 20(55 \%)$ & 0.7524 & $0.9(0-3)$ & 0 & 0.6185 \\
\hline & MACS & 1500 & 85 & $17 / 20(85 \%)$ & 0.0187 & $4.3(0-11)$ & 377.8 & 0.0003 \\
\hline \multirow[t]{4}{*}{2} & Control & 300 & 230 & 18/20 (90\%) & - & $11.5(0-27)$ & - & - \\
\hline & Centrifugation & 1500 & 413 & $20 / 20(100 \%)$ & 0.4872 & $20.7(2-46)$ & 80 & 0.0011 \\
\hline & Percoll & 1500 & 225 & 18/20 (90\%) & 1 & $11.3(0-23)$ & -1.7 & 0.8494 \\
\hline & MACS & 1500 & 482 & $20 / 20$ (100\%) & 0.4872 & $24.1(8-38)$ & 109.6 & $<0.0001$ \\
\hline \multirow[t]{4}{*}{3} & Control & 300 & 103 & 18/20 (80\%) & - & $5.2(0-20)$ & - & - \\
\hline & Centrifugation & 1500 & 0 & 0/20 (0\%) & $<0.0001$ & $0(0)$ & -100 & $<0.0001$ \\
\hline & Percoll & 1500 & 313 & 19/20 (95\%) & 0.3416 & $15.7(0-30)$ & 201.9 & 0.0002 \\
\hline & MACS & 1500 & 135 & $20 / 20(100 \%)$ & 0.106 & $6.8(1-14)$ & 30.8 & 0.2348 \\
\hline
\end{tabular}

\footnotetext{
* Significance compared to paired control

${ }^{\text {a }}$ Before concentration; blood volume in feeder $300 \mu \mathrm{L}$
} 

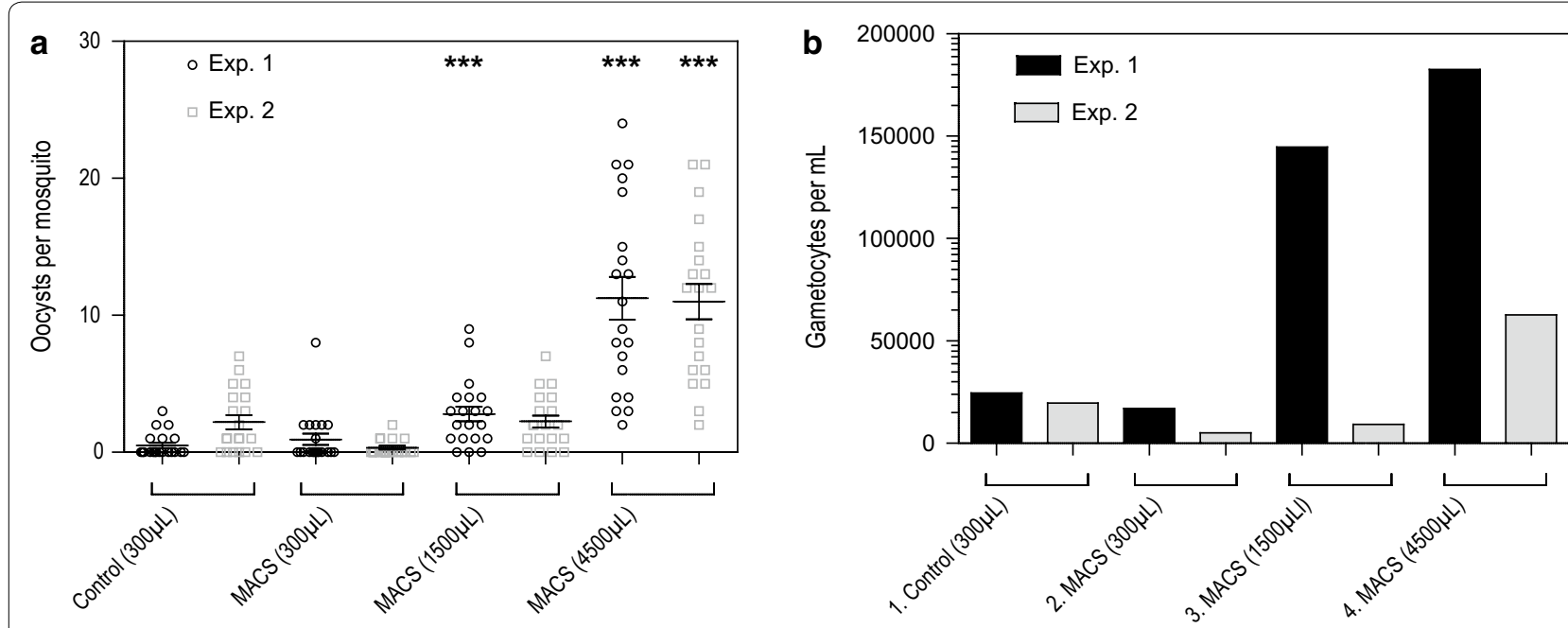

Fig. 2 The effect blood sample size on gametocyte concentration and infectivity by MACS. a The starting material of gametocyte-infected whole blood was $300 \mu \mathrm{L}$ for the controls, which was fed to mosquitoes directly. For the MACS, starting material was 300, 1500 and $4500 \mu \mathrm{L}$, respectively. Gametocytes from this starting material were concentrated, eluted and reconstituted in $400 \mu \mathrm{L}$ of uninfected blood, from which $300 \mu \mathrm{L}$ was fed to the mosquitoes. Vertical bars indicate mean with SEM. ${ }^{* *} \mathrm{p}<0.001$. b Gametocyte density per sample from feeding material measured by Pfs 25 qRT-PCR

mosquitoes, up to $70 \%$ of mosquitoes were infected using the 4500 and $9000 \mu \mathrm{L}$ samples (Fig. 3a). The total number of gametocytes per sample after purification was higher when a larger volume of blood was used and when the blood was run twice over the MACS (Fig. 3b). The material collected from the flow-through of the MACS prior to its removal from the magnet was tested in the qRTPCR. The flow-through contained approximately 5-9\% of the total gametocyte content of the start material that was not retained by the magnet. Follow-on experiments indicated that this loss could be reduced to $\sim 1 \%$ by running the blood twice over the MACS, thereby maximizing
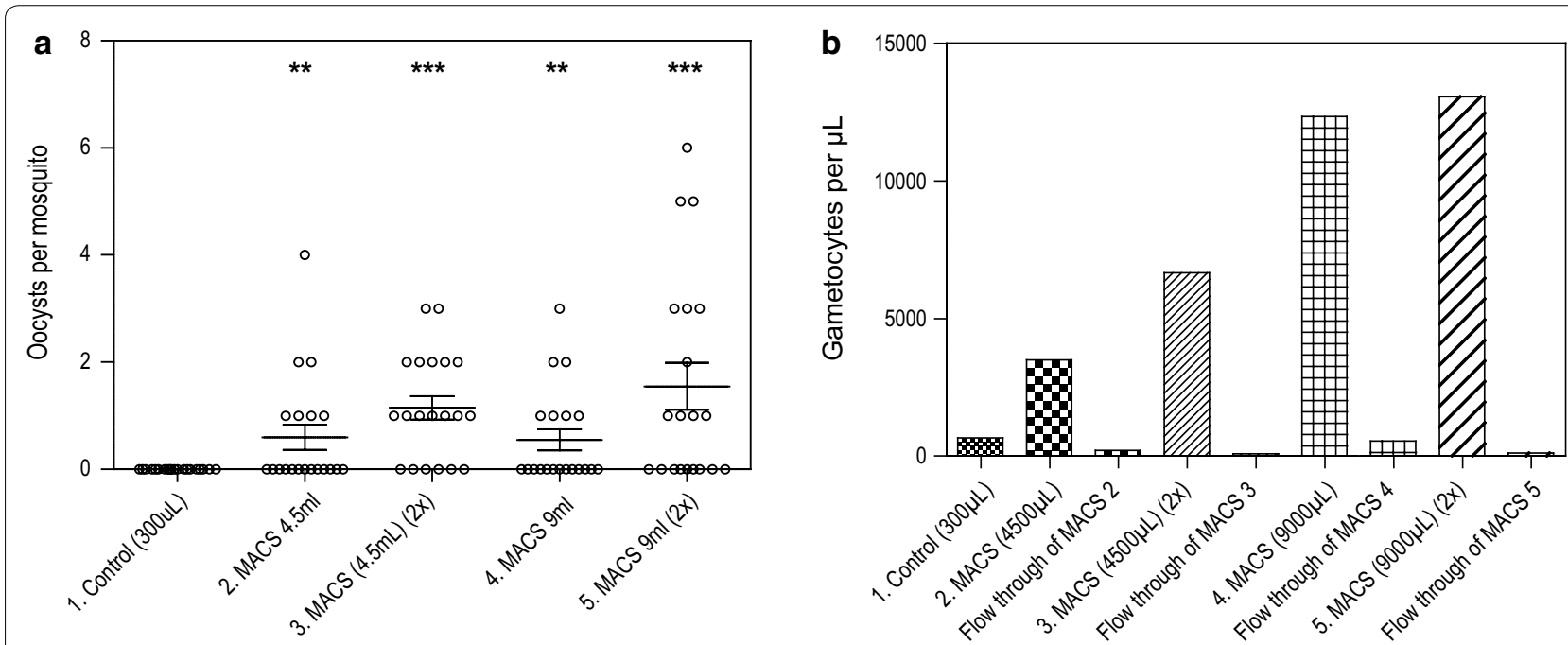

Fig. 3 Concentration and infectivity of low gametocyte densities after MACS. a $1300 \mu \mathrm{L}$ of gametocyte-infected whole blood from starting material, fed directly (control). $24500 \mu \mathrm{L}$ of gametocyte-infected whole blood from starting material added once over MACS column, eluted and reconstituted in $400 \mu \mathrm{L}$ of uninfected blood, from which $300 \mu \mathrm{L}$ was fed. $34500 \mu \mathrm{L}$ of gametocyte-infected whole blood from starting material run twice over MACS column, eluted and reconstituted in $400 \mu \mathrm{L}$ of uninfected blood, from which $300 \mu \mathrm{L}$ was fed. $49000 \mu \mathrm{L}$ of gametocyte-infected whole blood from starting material added once over MACS column, eluted and reconstituted in $400 \mu \mathrm{L}$ of uninfected blood, from which $300 \mu \mathrm{L}$ was fed. 5 $9000 \mu \mathrm{L}$ of gametocyte-infected whole blood from starting material run twice over MACS column, eluted and reconstituted in $400 \mu \mathrm{L}$ of uninfected blood, from which $300 \mu \mathrm{L}$ was fed. Vertical bars indicate mean with SEM. ${ }^{* *} p<0.01,{ }^{* * *} p<0.001$. b Gametocyte density per sample from feeding material measured by Pfs 25 qRT-PCR. Flow through of MACS represents the loss of gametocytes after MACS purification 
the likelihood of successful adherence of gametocytes to the magnetic column.

Gametocytes were titrated to assess mosquito infection rates after MACS at different gametocyte densities as determined by microscopy (Fig. 4a, b). The starting concentration and subsequent dilutions were either fed directly to mosquitoes or used for MACS gametocyte enrichment. Plotted are infection rates relative to the original gametocyte density. Control feeds used $300 \mu \mathrm{L}$ of gametocyte-infected whole blood versus $5000 \mu \mathrm{L}$ in the MACS gametocyte-enrichment feeds. A significant increase in the proportion of infected mosquitoes and oocyst density was found when using the MACS with the same gametocyte density, but a higher volume of gametocyte-infected blood. These increases in transmission resulted in a lower minimum gametocyte density to successfully measure transmission to mosquitoes.

Gametocyte densities were positively associated with infection rates $(r=0.6037, p \leq 0.0001)$ and mean oocyst numbers $(r=0.5968, p \leq 0.0001)$ in the general experiments as shown in Additional file 1: Figure S1.

\section{Discussion}

The current study successfully demonstrates that different concentration methods of $P$. falciparum gametocyteinfected whole blood samples enhance transmission in mosquito-feeding assays. The MACS gametocyte enrichment method showed an increase in oocyst intensity compared to control in all replicates. The MACS has been used previously for gametocyte synchronization and gametocyte detection in field studies [26]. This study shows that gametocytes remain infective after passage over a MACS column with densities as low as 1-100 gametocytes $/ \mu \mathrm{L}$ (Table 1 ).
Assessing the infectivity of low-density gametocytes is relevant for malaria control for areas with high prevalence of low-density infections [17, 27]. The stochastic nature of mosquito infections at low gametocyte densities complicates assessments of their transmissibility. An impractical number of mosquitoes needs to be examined to rule out infectivity with certainty. Whilst mosquito infection rates (and the intensity of infection in these mosquitoes) increase with increasing gametocyte density [28] a non-negligible fraction of high-density gametocyte infections appears to be sterile and unable to infect mosquitoes [3, 29]. It is increasingly recognized that the presence or density alone does not equal their infectiousness and direct assessments of the viability of gametocytes are essential [30, 31]. In epidemiological and intervention studies, it may thus be of relevance to determine whether gametocytes are capable of transmission regardless of their density. Sensitive mosquito feeding assays may be of particular relevance to study the dynamics of gametocyte infectivity in natural infections. Experimental infections suggests that infectivity may peak early in infections and gametocytes that are observed later in infections may be comparatively less infectious or non-infectious [32]. If the same applies to natural infections, this would be highly relevant since some gametocyte infections may thus be considered less important for malaria transmission. Similarly, clinical trials that specifically aim to induce gametocytes in volunteers may require an operationally attractive approach to enrich for gametocytes to increase the chances of successful mosquito infection and demonstrate the generation of viable gametocytes in these trials.

The MACS gametocyte enrichment method is simple, rapid and has been used extensively to concentrate gametocytes for purposes other than mosquito infection
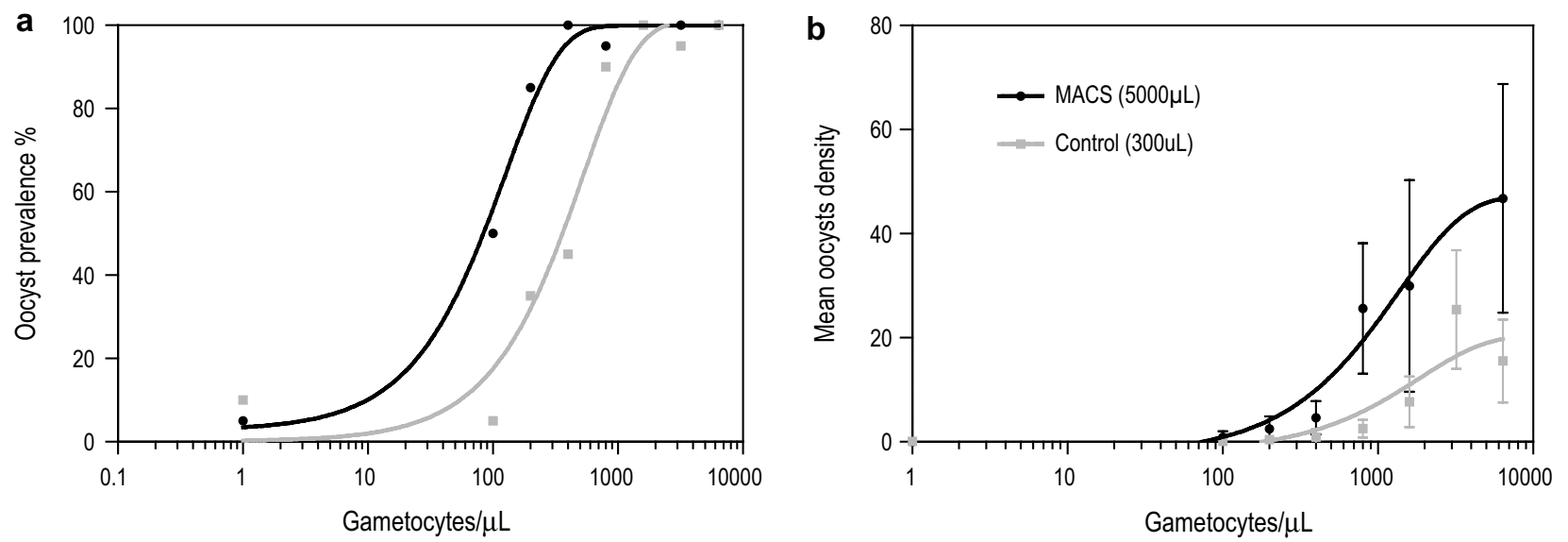

Fig. 4 Infectivity of different gametocyte densities after MACS versus control in a titration experiment. a Percentage of infected mosquitoes after titration experiment with MACS versus controls. b Number of oocysts per mosquito after titration experiment with MACS versus controls. Vertical bars indicate mean with SEM 
[23]. In the current experiments, MACS gave repeatedly high infection intensities compared to control and was, therefore, studied in more detail to test its ability to concentrate gametocytes from input material with low gametocyte concentrations. For this purpose, saturation of columns forms a concern in MACS procedures. The current study shows that the saturation point of the MACS was not reached when using a relatively large volume of $9000 \mu \mathrm{L}$ of blood with an estimated number of 9,000,000 gametocytes (at $1000 / \mu \mathrm{L})$. As previously shown, a reduction in flow rate through the MACS column could enhance the yield of Plasmodium berghei ookinetes [33], but no significant difference was observed when the flow rate was reduced by $\sim 1.5$ times, with a $23 \mathrm{G}$ needle compared to the $21 \mathrm{G}$ needle (Additional file 2: Figure S2). Nevertheless, further alteration of the flow rate may increase the efficiency of first pass column binding, and should therefore be investigated in future studies.

Previously, it has been reported that magnetic fields may affect parasite viability by compromising the growth of asexual P. falciparum forms [34]. This may relate to inhibition of polymerization of haem into haemozoin, and organelle damage due to the oscillation of haemozoin. In the current study, the magnetic field induced by the MACS appears to have limited impact on gametocyte fitness. Furthermore, no increase in infectivity was observed when concentrated gametocytes after MACS are brought back into culture for $24 \mathrm{~h}$, whilst the risk of contamination was considerable (unpublished findings). In addition, handling time, stress and temperature drops during sample transfer may also impact gametocyte infectivity. In the current study these factors appeared to have limited impact on gametocyte fitness and infectivity and the advantages of gametocyte concentration outweighed their potential detrimental effects.

No formal optimization of alternative gametocyteenrichment protocols was undertaken in this study. The current experiments therefore do not preclude that assay optimizations may increase transmission success of the tested enrichment procedures. Furthermore, the number of replicates was limited in the current study and the inevitable variation in transmission outcomes in standard membrane feeding assays with cultured gametocytes [35] warrants caution when comparing feeders within or between experiments. In addition, the lower efficiency of Percoll and standard centrifugation in the other experiments are most likely due to the low enrichment of gametocytes as reflected by the PCR results. When considering the other concentration methods evaluated, the standard centrifugation was indeed attractive because of its simplicity. However, the absence of an unequivocal visual cell layer made it difficult to reproducibly obtain a well-defined layer of gametocytes. An additional potential disadvantage of density-dependent centrifugation is that it is unable to separate gametocytes from host white blood cells [21, 22]. As host white blood cells can interfere with the success rate of transmission in membrane-feeding assays, such cell contamination is undesirable and will require another step of filtration [36-38]. Trang et al. shows that the MACS purification removes approximately $94 \%$ of the white blood cells, in contrast to $0 \%$ white blood cell removal after Percoll gradient, or other density gradient separation such as Nycodenz [39].

\section{Conclusions}

The current study demonstrates that gametocyte purification by MACS concentrates gametocytes present at low density in whole blood samples whilst retaining their infectivity in membrane-feeding assays. Whilst current conclusions were based on a limited number of replicates, they formed the evidence base for the selection of a gametocyte enrichment method for a controlled human malaria infection study (CHMI) that aimed to induce (low-densities) gametocytes. In general, the methodology presented here may facilitate the use of blood samples from naturally or experimentally infected individuals to evaluate human host infectivity and malaria transmission-blocking interventions.

\section{Additional files}

\begin{abstract}
Additional file 1: Figure S1. Association between gametocyte densities and oocyst-prevalence and density. A. Overview of gametocyte densities and infection rates of experiments of Figs. 1, 2, 3, 4 and Additional file 2: Figure S2. B. Overview of gametocyte densities and mean oocysts of experiments of Figs. 1, 2, 3, 4 and Additional file 2: Figure S2.

Additional file 2: Figure S2. Effect of different flow-rate MACS on gametocyte infectivity. Number of oocysts per mosquito after membranefeeding assay (MIDI cages). $1600 \mu \mathrm{L}$ of gametocyte-infected whole blood from starting material, fed directly. $29000 \mu \mathrm{L}$ of gametocyte-infected whole blood from starting material added once over MACS column with $23 \mathrm{G}$ needle, eluted and reconstituted in $600 \mu \mathrm{L}$ of uninfected blood before feed. $39000 \mu \mathrm{L}$ of gametocyte-infected whole blood from starting material added once over MACS column with $21 \mathrm{G}$ needle, eluted and reconstituted in $600 \mu \mathrm{L}$ of uninfected blood before feed. Vertical bars indicate mean with SEM.
\end{abstract}

\begin{abstract}
Abbreviations
SMFA: standard membrane-feeding assay; CHMI: controlled human malaria infection; MACS: magnetic cell sorting; qRT-PCR: quantitative reverse transcription PCR.

\section{Authors' contributions}

IJR, WJRS, MV, GG, RS, WG, TB, and RWS conceived the study and participated in its design. KL performed the qRT-PCR. All authors participated in the data analysis and writing of the manuscript. All authors read and approved the final manuscript.
\end{abstract}

\section{Acknowledgements}

We would like to thank Matthijs M Jore at the Radboud University Medical Centre for his advice and input in the experiments. 


\section{Competing interests}

The authors declare that they have no competing interests.

\section{Ethics approval and consent to participate}

Not applicable.

\section{Funding}

WJRS, KL and TB are supported by a fellowship from the European Research Council (ERC-2014-StG 639776).

\section{Publisher's Note}

Springer Nature remains neutral with regard to jurisdictional claims in published maps and institutional affiliations.

Received: 6 June 2017 Accepted: 28 July 2017

Published online: 05 August 2017

\section{References}

1. von Seidlein L, Dondorp A. Fighting fire with fire: mass antimalarial drug administrations in an era of antimalarial resistance. Expert Rev Anti Infect Ther. 2015;13:715-30.

2. Alonso PL, Brown G, Arevalo-Herrera M, Binka F, Chitnis C, Collins F, et al. A research agenda to underpin malaria eradication. PLoS Med. 2011:8:e1000406.

3. Bousema T, Dinglasan RR, Morlais I, Gouagna LC, van Warmerdam T, Awono-Ambene $\mathrm{PH}$, et al. Mosquito feeding assays to determine the infectiousness of naturally infected Plasmodium falciparum gametocyte carriers. PLOS ONE. 2012;7:e42821.

4. Bousema T, Drakeley C. Epidemiology and infectivity of Plasmodium falciparum and Plasmodium vivax gametocytes in relation to malaria control and elimination. Clin Microbiol Rev. 2011:24:377-410.

5. Boudin C, Olivier M, Molez JF, Chiron JP, Ambroise-Thomas P. High human malarial infectivity to laboratory-bred Anopheles gambiae in a village in Burkina Faso. Am J Trop Med Hyg. 1993;48:700-6.

6. Haji H, Smith T, Charlwood JD, Meuwissen JH. Absence of relationships between selected human factors and natural infectivity of Plasmodium falciparum to mosquitoes in an area of high transmission. Parasitology. 1996:113(Pt 5):425-31.

7. Farnert A, Snounou G, Rooth I, Bjorkman A. Daily dynamics of Plasmodium falciparum subpopulations in asymptomatic children in a holoendemic area. Am J Trop Med Hyg. 1997;56:538-47.

8. Mharakurwa S, Kumwenda T, Mkulama MA, Musapa M, Chishimba S, Shiff CJ, et al. Malaria antifolate resistance with contrasting Plasmodium falciparum dihydrofolate reductase (DHFR) polymorphisms in humans and Anopheles mosquitoes. Proc Natl Acad Sci USA. 2011;108:18796-801.

9. Lambrechts L, Halbert J, Durand P, Gouagna LC, Koella JC. Host genotype by parasite genotype interactions underlying the resistance of anopheline mosquitoes to Plasmodium falciparum. Malar J. 2005:4:3.

10. Targett G, Drakeley C, Jawara M, von Seidlein L, Coleman R, Deen J, et al. Artesunate reduces but does not prevent posttreatment transmission of Plasmodium falciparum to Anopheles gambiae. J Infect Dis. 2001;183:1254-9.

11. Hallett RL, Dunyo S, Ord R, Jawara M, Pinder M, Randall A, et al. Chloroquine/sulphadoxine-pyrimethamine for gambian children with malaria: transmission to mosquitoes of multidrug-resistant Plasmodium falciparum. PLoS Clin Trials. 2006;1:e15.

12. Robert $\mathrm{V}$, Read AF, Essong J, Tchuinkam T, Mulder B, Verhave JP. Effect of gametocyte sex ratio on infectivity of Plasmodium falciparum to Anopheles gambiae. Trans R Soc Trop Med Hyg. 1996;90:621-4.

13. Mitri C, Thiery I, Bourgouin C, Paul RE. Density-dependent impact of the human malaria parasite Plasmodium falciparum gametocyte sex ratio on mosquito infection rates. Proc Biol Sci. 2009:276:3721-6.

14. Bousema T, Sutherland CJ, Churcher TS, Mulder B, Gouagna LC, Riley EM, et al. Human immune responses that reduce the transmission of Plasmodium falciparum in African populations. Int J Parasitol. 2011;41:293-300.

15. Graves PM, Doubrovsky A, Sattabongkot J, Battistutta D. Human antibody responses to epitopes on the Plasmodium falciparum gametocyte antigen PFS 48/45 and their relationship to infectivity of gametocyte carriers. Am J Trop Med Hyg. 1992;46:711-9.

16. Michel K, Kafatos FC. Mosquito immunity against Plasmodium. Insect Biochem Mol Biol. 2005;35:677-89.

17. Okell LC, Bousema T, Griffin JT, Ouedraogo AL, Ghani AC, Drakeley CJ. Factors determining the occurrence of submicroscopic malaria infections and their relevance for control. Nat Commun. 2012;3:1237.

18. Pasay CJ, Rockett R, Sekuloski S, Griffin P, Marquart L, Peatey C, et al. Piperaquine monotherapy of drug-susceptible Plasmodium falciparum infection results in rapid clearance of parasitemia but is followed by the appearance of gametocytemia. J Infect Dis. 2016;214:105-13.

19. Griffin P, Pasay C, Elliott S, Sekuloski S, Sikulu M, Hugo L, et al. Safety and reproducibility of a clinical trial system using induced blood stage Plasmodium vivax infection and its potential as a model to evaluate malaria transmission. PLoS Negl Trop Dis. 2016;10:e0005139.

20. Ponnudurai T, Lensen AH, Meis JF, Meuwissen JH. Synchronization of Plasmodium falciparum gametocytes using an automated suspension culture system. Parasitology. 1986;93(Pt 2):263-74.

21. Andrysiak PM, Collins WE, Campbell GH. Concentration of Plasmodium ovale- and Plasmodium vivax-infected erythrocytes from nonhuman primate blood using Percoll gradients. Am J Trop Med Hyg. 1986;35:251-4.

22. Ihalamulla RL, Mendis KN. Plasmodium vivax: isolation of mature asexual stages and gametocytes from infected human blood by colloidal silica (Percoll) gradient centrifugation. Trans R Soc Trop Med Hyg. 1987;81:25-8.

23. Karl S, Davis TM, St-Pierre TG. A comparison of the sensitivities of detection of Plasmodium falciparum gametocytes by magnetic fractionation, thick blood film microscopy, and RT-PCR. Malar J. 2009;8:98.

24. Paul F, Roath S, Melville D, Warhurst DC, Osisanya JO. Separation of malaria-infected erythrocytes from whole blood: use of a selective highgradient magnetic separation technique. Lancet. 1981;2:70-1.

25. Pett H, Goncalves BP, Dicko A, Nebie I, Tiono AB, Lanke K, et al. Comparison of molecular quantification of Plasmodium falciparum gametocytes by Pfs 25 qRT-PCR and QT-NASBA in relation to mosquito infectivity. Malar J. 2016;15:539.

26. Saliba KS, Jacobs-Lorena M. Production of Plasmodium falciparum gametocytes in vitro. Methods Mol Biol. 2013;923:17-25.

27. Slater HC, Ross A, Ouedraogo AL, White LJ, Nguon C, Walker PG, et al. Assessing the impact of next-generation rapid diagnostic tests on Plasmodium falciparum malaria elimination strategies. Nature. 2015:528:594-101.

28. Da DF, Churcher TS, Yerbanga RS, Yameogo B, Sangare I, Ouedraogo JB, et al. Experimental study of the relationship between Plasmodium gametocyte density and infection success in mosquitoes; implications for the evaluation of malaria transmission-reducing interventions. Exp Parasitol. 2015;149:74-83.

29. Churcher TS, Bousema T, Walker M, Drakeley C, Schneider P, Ouedraogo $\mathrm{AL}$, et al. Predicting mosquito infection from Plasmodium falciparum gametocyte density and estimating the reservoir of infection. Elife. 2013:2:e00626

30. Karunajeewa HA, Mueller I. How important is gametocyte clearance after malaria therapy? BMC Med. 2016;14:93.

31. White NJ, Ashley EA, Recht J, Delves MJ, Ruecker A, Smithuis FM, et al. Assessment of therapeutic responses to gametocytocidal drugs in Plasmodium falciparum malaria. Malar J. 2014;13:483.

32. Johnston GL, Smith DL, Fidock DA. Malaria's missing number: calculating the human component of RO by a within-host mechanistic model of Plasmodium falciparum infection and transmission. PLoS Comput Biol. 2013;9:e1003025

33. Carter V, Cable HC, Underhill BA, Williams J, Hurd H. Isolation of Plasmodium berghei ookinetes in culture using Nycodenz density gradient columns and magnetic isolation. Malar J. 2003;2:35.

34. Feagin JE, Wurscher MA, Ramon C, Lai HC. Magnetic fields and malaria. In: Holick MF, Jung EG, editors. Biologic effects of light. Hingham: Academic Publishers; 1999

35. Churcher TS, Blagborough AM, Delves M, Ramakrishnan C, Kapulu MC, Williams AR, et al. Measuring the blockade of malaria transmissionan analysis of the standard membrane feeding assay. Int J Parasitol. 2012:42:1037-44.

36. Lensen A, Mulder L, Tchuinkam T, Willemsen L, Eling W, Sauerwein R. Mechanisms that reduce transmission of Plasmodium falciparum malaria in semiimmune and nonimmune persons. J Infect Dis. 1998;177:1358-63. 
37. Healer J, Graszynski A, Riley E. Phagocytosis does not play a major role in naturally acquired transmission-blocking immunity to Plasmodium falciparum malaria. Infect Immun. 1999;67:2334-9.

38. Stone WJ, Dantzler KW, Nilsson SK, Drakeley CJ, Marti M, Bousema T, et al. Naturally acquired immunity to sexual stage P. falciparum parasites. Parasitology. 2016;143:187-98.

39. Trang DT, Huy NT, Kariu T, Tajima K, Kamei K. One-step concentration of malarial parasite-infected red blood cells and removal of contaminating white blood cells. Malar J. 2004;3:7

40. Stone W, Sawa P, Lanke K, Rijpma S, Oriango R, Nyaurah M, Osodo P, Osoti V, Mahamar A, Diawara H, Woestenenk R, Graumans W, van de
Vegte-Bolmer M, Bradley J, Chen I, Brown J, Siciliano G, Alano P, Gosling R, Dicko A, Drakeley C, Bousema T. A molecular assay to quantify male and female plasmodium falciparum gametocytes: results from 2 randomized controlled trials using primaquine for gametocyte clearance. J Infect Dis. 2017. doi:10.1093/infdis/jix237

\section{Submit your next manuscript to BioMed Central and we will help you at every step:}

- We accept pre-submission inquiries

- Our selector tool helps you to find the most relevant journal

- We provide round the clock customer support

- Convenient online submission

- Thorough peer review

- Inclusion in PubMed and all major indexing services

- Maximum visibility for your research

Submit your manuscript at www.biomedcentral.com/submit 